\title{
INVENTARIO DE LOS RESIDUOS SÓLIDOS GENERADOS EN LAS EMPRESAS ANTIOQUEÑAS PERTENECIENTES A LOS PRINCIPALES SECTORES ECONÓMICOS DEL DEPARTAMENTO
}

\author{
Dora Ángela Hoyos \\ PhD. Ciencias Químicas \\ Directora de la Corporación Académica \\ Ciencias Básicas Biomédicas \\ Universidad de Antioquia \\ dorangel@udea.edu.co
}

\author{
Margarita Hincapié \\ PhD. Ciencias Químicas \\ Docente tiempo completo \\ Universidad de Medellín \\ mhincapie@udem.edu.co
}

\author{
Juan Miguel Marín \\ PhD. Ciencias Químicas \\ Docente Departamento de \\ Ingeniería Química \\ Universidad de Antioquia \\ jmmarin@udea.edu.co
}

\author{
Lina Marcela Jiménez \\ Ingeniera Ambiental \\ Docente de cátedra \\ Universidad de San Buenaventura \\ marcelaj13@hotmail.com
}

\author{
Germán Mauricio Valencia \\ Especialista en SIG \\ Docente tiempo completo \\ Universidad de San Buenaventura \\ especialización.sig@usbmed.edu.co
}

(Tipo de Artículo: Investigación. Recibido el 15/10/2012. Aprobado el 24/05/2013)

\section{RESUMEN}

Este artículo muestra los resultados de una investigación sobre la producción de residuos sólidos generados por las empresas Antioqueñas. Investigación que fue desarrollada por la Universidad de San Buenaventura, la Universidad de Medellín y la Universidad de Antioquia dentro del programa Expedición Antioquia 2013. Para lo cual, se evaluó la producción de residuos generados en empresas pertenecientes a las actividades económicas; agricultura, ganadería, caza y silvicultura; Explotación de minas y canteras; Industrias manufactureras y Construcción.

\begin{abstract}
El propósito del estudio fue conocer la tipología, cantidad e impacto ambiental de los residuos generados en el departamento en los sectores seleccionados, constituyéndose en una base para futuras investigaciones, orientadas a determinar el potencial aprovechamiento de los residuos para la elaboración de nuevos productos. La zona de estudio corresponde al 80,8\% de la superficie del departamento de Antioquia, subregiones, Bajo Cauca, Nordeste, Norte, Oriente, Suroeste, Urabá y Valle de Aburrá; las cuales concentran las zonas industriales, mineras y agropecuarias más representativas del departamento, además de ser subregiones altamente pobladas con un crecimiento en infraestructura, representado en un sector de construcción fortalecido.
\end{abstract}

La metodología utilizada fue la construcción de bases de datos de las empresas de los sectores seleccionados para posteriormente obtener información de la generación de residuos sólidos por medio de encuestas realizadas por un aplicativo web, personal o telefónicamente.

Palabras clave

Inventario de residuos, residuos industriales, residuos sólidos, Expedición Antioquia 2013, Aprovechamiento de residuos.

\section{INVENTORY OF SOLID WASTE GENERATED BY COMPANIES BASED IN ANTIOQUIA THAT BELONG TO PRIMARY ECONOMIC SECTORS}

\begin{abstract}
This article shows the results of a research on the production of solid waste generated by companies based in Antioquia. This Research was conducted by the University of San Buenaventura, University of Medellin and the University of Antioquia in the program called Antioquia Expedition 2013. For this purpose, we evaluated the production of waste generated by companies in economic activities such as agriculture, cattle ranching, hunting and forestry, mining and quarry exploitation, manufacturing and construction.

The purpose of the study was to determine the type, quantity and environmental impact of the waste generated in Antioquia in the selected sectors, constituting itself into a basis for further investigations aimed in determining the potential use of waste for creating new products. The territory analyzed corresponds to $80.8 \%$ of the area belonging to the department of Antioquia, subregions, Bajo Cauca, North-east, North, East, South west, Urabá and Valle de Aburrá, where are concentrated the most significant industrial, mining and farming areas of the department, besides being highly populated subregions with growth in infrastructure, represented in a stronger construction sector.
\end{abstract}

The methodology used was the development of databases of companies belonging to the selected sectors to subsequently get information on solid waste generation through surveys conducted by means of a web application, face-to-face or by telephone.

\section{Keywords}

Inventory of waste, industrial waste, solid waste, Antioquia Expedition 2013, use of waste. 


\title{
INVENTAIRE DES DÉCHETS SOLIDES GÉNERÉS DANS LES ENTREPRISES D'ANTIOQUIA-COLOMBIE QUI APPARTIENNENT AUX PRINCIPAUX SECTEURS ÉCONOMIQUES DU DÉPARTEMENT
}

\begin{abstract}
Résumé
Cet article présent les résultats d'une recherche au sujet de la production de déchets solides par les entreprises d'Antioquia. La recherche a été réalisée par l'Université de San Buenaventura, l'Université de Medellín et l'Université d'Antioquia au sein du programme Expédition Antioquia 2013. On a évalué la production de déchets génères dans entreprises qui appartiennent aux suivants activités économiques: agriculture, l'élevage du bétail, la chasse et la sylviculture, l'exploitation des mines et des carrières, des industries de manufacture et de la construction.
\end{abstract}

Le but de la recherche est de connaitre la typologie, quantité et effet environnemental des déchets produits dans le département d'Antioquia dans les secteurs déjà mentionnés, en se constituant comme une base pour futurs recherches, dirigées vers la détermination de l'utilisation potentiel des déchets pour l'élaboration de nouveaux produits. La zone étudié inclut le 80.8 pour cent de la surface du département d'Antioquia, sous-régions, bas Cauca, le Nord Est, le Nord, l'Est, le Sud Ouest, l'Urabá et le Valle de Aburrá; où sont concentrés las zones industrielles, miniers et agricoles les plus représentatives du département, en plus d'être des sous-régions hautement peuplés avec un croissance dans infrastructure, qui est représenté par un secteur de construction fortifié.

La méthodologie utilisée a été la construction des bases de données des entreprises des secteurs sélectionnés pour obtenir l'information de la production de déchets solides en utilisant des enquêtes réalisés au moyen d'un logiciel web, en personne o téléphoniquement.

Mots-clés. Inventaire de déchets, déchets industriels, déchets solides, Expédition Antioquia 2013, utilisation des déchets. 


\section{INTRODUCCIÓN}

El aumento en la densidad poblacional y la creciente industrialización a nivel mundial, han puesto al ser humano como uno de los principales causantes de pérdida de biodiversidad; por cambios de hábitat, cambio climático, sobre-explotación de recursos y contaminación [1], es por esto que el tema ambiental debe ir paralelo al crecimiento industrial. Dentro de las principales preocupaciones de la planeación ambiental se encuentran los residuos sólidos y dentro de éstos son los residuos industriales los que presentan mayor problemática, debido a las grandes cantidades de generación y la composición fisicoquímica, que dificulta su eliminación por procesos naturales.

Una adecuada gestión de los residuos es importante para disminuir el consumo de recursos, incrementar la eficiencia de un proceso y reducir los costos ambientales asociados al tratamiento y disposición final; una óptima gestión de los residuos sólidos debe incluir la minimización, aprovechamiento, recuperación, reutilización y transformación mediante el uso de tecnologías alternas, además de capacitación, educación, divulgación, participación ciudadana y cooperación interinstitucional [2], [3]; sin embargo, en Antioquia la gestión de los residuos sólidos se ha enfocado principalmente en la prestación del servicio público de aseo en las etapas de recolección y transporte de residuos hasta el sitio de disposición final, dejando de lado los demás aspectos [4].

Con el fin de buscar alternativas de disminución de residuos y/o desarrollar tecnologías limpias y buenas prácticas que permitan la prevención y minimización de residuos en el departamento de Antioquia, se realizó un diagnóstico que permitiera conocer el tipo y cantidad de residuos producidos por actividad económica; para posteriormente determinar su composición y poder así diseñar estrategias de aprovechamiento. Este diagnóstico se llevó a cabo en la primera fase del proyecto "Residuos sólidos potencialmente aprovechables en las empresas antioqueñas para la aplicación en nuevos productos", en el eje de Ingeniería, Recursos y Desarrollo del programa Expedición Antioquia 2013, el cual se realizó como una conmemoración al bicentenario de independencia del departamento, apoyándose en la investigación científica como herramienta para contribuir al mejoramiento de las condiciones de vida y al desarrollo tecnológico del departamento, a partir del adecuado aprovechamiento, manejo, uso y preservación de los recursos naturales. El proyecto fue ejecutado por la Universidad de San Buenaventura, la Universidad de Medellín y la Universidad de Antioquia; con la participación del Grupo de Ingeniería y Gestión Ambiental (GIGA), el Grupo de Procesos Fisicoquímicos aplicados (PFA) de la Universidad de Antioquia, el Grupo de Investigaciones y Mediciones Ambientales (GEMA) de la Universidad de Medellín; y el Grupo de Investigación en Modelamiento y Simulación Computacional (GIMSC) de la Universidad de San Buenaventura.
Para la realización del proyecto se tuvieron en cuenta las bases de datos de las empresas Antioqueñas suministradas por diferentes entidades, esta información fue la base para identificar la principal actividad económica de cada subregión, luego por medio de un análisis estadístico se seleccionaron las actividades económicas a intervenir (agrícola, minero, manufacturero y construcción) para la realización del diagnóstico. A las empresas pertenecientes a las actividades económicas seleccionadas por subregión, se les aplicó una encuesta para obtener información primaria de generación y manejo de residuos; dando como resultado la georeferenciación de las empresas de los sectores seleccionados, así como la georeferenciación de los residuos sólidos reportados por éstas.

\section{METODOLOGÍA}

Para el inventario de residuos sólidos industriales se realizó una búsqueda de información para conformar una base de datos sobre las actividades económicas y tipos de empresas del departamento con su respectiva cartografía; lo que pudo lograrse a través de contactos con alcaldías, autoridades ambientales, cámaras de comercio, agremiaciones y asociaciones de empresarios.

En la base de datos consolidada se agruparon las empresas del departamento por actividades económicas a través de la clasificación CIIU (Clasificación Industrial Internacional Uniforme de Actividades Económicas) versión 3. Esta es una estructura jerarquizada que codifica las actividades económicas en diferentes niveles cuyo objetivo principal es proporcionar una nomenclatura única para obtener homogeneidad en las estadísticas sectorizadas por actividad económica y poder hacer comparaciones nacionales e internacionales [5]. Esta clasificación está compuesta por una letra y cuatro dígitos, la letra representa el Nivel e indica la actividad económica, los primeros dos dígitos indican el sector productivo y los segundos dos dígitos indican el subsector productivo.

El análisis de las actividades descritas en la clasificación CIIU, dio como resultado la selección de los niveles y sectores objeto de estudio, excluyendo actividades económicas que generaran principalmente residuos denominados como comunes (papel, cartón, residuos de alimentos, entre otros) y que además cuentan con un manejo claramente establecido por la normativa existente, tanto para su presentación, transporte y disposición final como para su aprovechamiento.

\subsection{Definición del área de estudio}

Se aplicó la técnica ANOVA (Análisis de Varianza), el test de rangos múltiples, para determinar las subregiones en las cuales predominaba las actividades económicas seleccionadas, este análisis se hizo por subregión, teniendo en cuenta los municipios y el número de empresas asentadas en ellos, además se relacionaron las subregiones buscando contrastes en cada una de ellas. 


\subsection{Divulgación del proyecto en las subregiones}

Para la divulgación del proyecto se realizaron diferentes actividades como: entrevistas radiales en varios municipios de las subregiones, boletines de prensa en periódicos reconocidos de la región, visita a agremiaciones de empresarios y entidades que tienen contacto directo con estos y socializaciones en las subregiones seleccionadas.

Tanto en entrevistas como en boletines se explicó el proyecto y la forma en la cual se realizaría el inventario de residuos. El contacto con agremiaciones buscó la difusión del proyecto por medio de correos masivos o visitas personalizadas a sus asociados para presentar el proyecto y obtener información de los residuos generados por ellos. En las socializaciones, se seleccionaron los municipios según concentración industrial y ubicación estratégica para el desplazamiento de los empresarios, en el Valle de Aburrá se realizó la socialización por medio de agremiaciones y asociaciones, debido al gran número de empresas ubicadas en la subregión.

\subsection{Recolección de datos}

Se aplicó una encuesta para obtener datos específicos sobre los residuos generados en las principales actividades económicas del Departamento. Para el diseño de la encuesta se tuvo en cuenta la información básica de la empresa, los procesos productivos, cantidad de residuos generados por tipo y manejo actual o tratamiento dado a los residuos, además del grado de organización al interior de la empresa en el tema de residuos y disponibilidad de la empresa para participar en la fase investigativa del proyecto.

Se hizo una encuesta para cada actividad económica y se realizó la prueba piloto durante las presentaciones del proyecto en los diferentes municipios para evaluar la correcta formulación de las preguntas, su grado de validez y la confiabilidad de los datos que serían recolectados; lo que permitió posteriormente, realizar los ajustes necesarios en la encuesta.

Considerando las características del estudio y la información disponible, la encuesta fue aplicada como un censo y fue enviada por correo certificado y por correo electrónico, a través de la compañía de encuesta electrónicas e-encuesta.

\subsection{Análisis de datos}

Los datos obtenidos se tabularon para determinar los residuos predominantes en el departamento y georeferenciarlos. Posteriormente se determinó la importancia de los impactos ambientales adaptando las metodologías de evaluación de impacto ambiental de Arboleda y Coneza [6], [7], a los atributos considerados como prioritarios para la identificación de los residuos más impactantes. Los atributos se describen a continuación:

Carácter (CA): Califica el impacto si es positivo o negativo.
Positivo $=1$

Negativo $=-1$

Generación (GE): Considera la cantidad producida del residuo y toma valores entre 1 y 15 , tomando el mayor valor para la mayor generación establecida (Tabla 1)

Tabla 1. Valor asignado por cantidad de residuos generada

\begin{tabular}{|l|l|c|}
\hline \multicolumn{1}{|c|}{ Generación } & \multicolumn{1}{c|}{ Rango } & Valor \\
\hline 1 ton/año a 25 ton/año & Muy baja & 3 \\
\hline 26 ton/año a 100 ton/año & Baja & 6 \\
\hline 101 ton/año a 250 ton/año & Media & 9 \\
\hline 251 ton/año a 500 ton/año & Alta & 12 \\
\hline Mayor a 500 ton/año & Muy alta & 15 \\
\hline
\end{tabular}

Extensión (EX): Se refiere a la incidencia del impacto en función del número de fuentes generadoras, en este caso depende del número de empresas que reportan el residuo de interés, su valoración es entre 1 y 15 (Tabla 2)

Tabla 2. Valor asignado por cantidad de empresas que generan el residuo

\begin{tabular}{|l|l|c|}
\hline \multicolumn{1}{|c|}{ Extensión } & \multicolumn{1}{c|}{ Rango } & Valor \\
\hline 0-5 empresas & Puntual & 3 \\
\hline 6-10 empresas & Parcial & 6 \\
\hline 11-15 empresas & Media & 9 \\
\hline 16-20 empresas & Extensa & 12 \\
\hline Mayor a 20 empresas & Muy extensa & 15 \\
\hline
\end{tabular}

Periodicidad (PE): Corresponde a la temporalidad en la generación del residuo, evalúa si se da de manera continua, periódica u ocasional. Se valora entre 1 y 5 (Tabla 3).

Tabla 3. Valoración asignada por la periodicidad en la generación del residuo

\begin{tabular}{|l|c|}
\hline Periodicidad & Valor \\
\hline Ocasional & 1 \\
\hline Periódica & 3 \\
\hline Continua & 5 \\
\hline
\end{tabular}

Reversibilidad (RV): Evalúa el tiempo de respuesta del medio para volver al estado natural superando el escenario más crítico de disposición del residuo. En esta valoración se debe considerar la peligrosidad, persistencia y daño que pueda causar la liberación del residuo al ambiente. Se valora entre 1 y 5 (Tabla 4)

Tabla 4. Valor asignado por el tiempo de respuesta del medio al impacto del residuo

\begin{tabular}{|l|c|}
\hline \multicolumn{1}{|c|}{ Reversibilidad } & Valor \\
\hline $\begin{array}{l}\text { Se supera en corto plazo } \\
\text { (menos de 1 año) }\end{array}$ & 1 \\
\hline $\begin{array}{l}\text { Se supera en mediano plazo } \\
\text { (entre 1 y 5 años) }\end{array}$ & 3 \\
\hline Irreversible & 5 \\
\hline
\end{tabular}

Manejo actual (MC): Considera las acciones desarrolladas en el contexto local para el aprovechamiento del residuo y la disminución de sus impactos. Se da una mayor importancia a los residuos que aún no tienen ningún tipo de alternativa de manejo 
diferente a la disposición final. Su valoración es entre 1 y 10 (Tabla 5)

Tabla 5. Valor asignado dependiendo del grado de aprovechamiento del residuo

\begin{tabular}{|l|c|}
\hline \multicolumn{1}{|c|}{ Manejo actual } & Valor \\
\hline Aprovechamiento total & 2 \\
\hline Aprovechamiento parcial & 6 \\
\hline No se aprovecha & 10 \\
\hline
\end{tabular}

Efecto (EF): Determina si las manifestaciones del impacto son consecuencia directa 0 indirecta del residuo de interés (Tabla 6 ).
Tabla 6. Valor asignado por el efecto del residuo

\begin{tabular}{|l|c|}
\hline \multicolumn{1}{|c|}{ Efecto } & Valor \\
\hline Indirecto & 1 \\
\hline Directo & 5 \\
\hline
\end{tabular}

Para la valoración de todos los atributos inicialmente se determina si hay afectación al recurso, en caso de no presentar afectación, el valor asignado es cero.

Importancia del impacto (I): estará dada por la siguiente expresión (1):

$I=C A^{\star}\left(3^{\star} G E+2^{\star} E X+P E+R V+M C+E F\right)$

De acuerdo a la valoración del impacto, estos se clasificaron según su grado de importancia (Tabla 7)

Tabla 7. Valoración del Impacto Ambiental

\begin{tabular}{|l|l|}
\hline ESCALA DE VALORACIÓN & IMPORTANCIA DEL IMPACTO \\
\hline Valor superior $a+100$ & Impacto benéfico importante \\
\hline Valor inferior $a+100$ & Impacto benéfico de importancia moderada \\
\hline Valores entre 0 y -120 & $\begin{array}{l}\text { Impacto irrelevante o compatible con el } \\
\text { medio }\end{array}$ \\
\hline Valores entre -120 y -240 & Impacto de Importancia Moderada \\
\hline Valores entre -240 y -360 & Impacto de Importancia Media \\
\hline Valores entre -360 y -480 & Impacto de Importancia Severa \\
\hline Valores por encima de -480 & Impacto de Importancia Crítica \\
\hline
\end{tabular}

Esta valoración tiene como finalidad priorizar investigaciones futuras sobre aquellos residuos que presenten un mayor impacto ambiental.

\section{RESULTADOS Y DISCUSIÓN}

Para la realización del inventario de residuos industriales, se seleccionaron 4 de las 17 actividades económicas, ésta selección buscaba intervenir actividades que fueran representativas en el departamento, a la vez que la generación de los residuos en ellas se diera de una manera significativa. Los niveles seleccionados se muestran en la Tabla 8, con la cantidad de subsectores asociados a ellas.

Con la información suministrada por las diferentes entidades, se consolidó una base de datos de 16.778 empresas de los sectores seleccionados; para la realización del inventario de residuos sólidos se excluyeron de la base de datos las empresas ubicadas en las subregiones occidente y magdalena medio, subregiones no incluidas en el estudio por presentar un número de empresas no significativo en comparación con las demás subregiones, con los datos restantes se realizó un análisis de varianzas, test de rangos múltiples y se seleccionaron a su vez los subsectores a intervenir por subregión, dando como resultado un total de 13.584 empresas para el estudio (Tabla 9), de estas empresas algunas presentaban errores en la dirección, se habían trasladado o dejaron de existir, por lo que la encuesta fue aplicada a las 12.054 empresas restantes las cuales pueden ser observadas en la Figura 1, donde se muestra la distribución geográfica de las empresas en el departamento de Antioquia.

Tabla 8. Actividades Económicas seleccionadas

\begin{tabular}{|c|l|c|}
\hline \multirow{2}{*}{ Niveles } & \multicolumn{1}{|c|}{ Descripción (Actividad Económica) } & $\begin{array}{c}\text { Número de Subsectores } \\
\text { Productivos adscritos a cada } \\
\text { Nivel }\end{array}$ \\
\hline A & Agricultura, ganadería, caza y silvicultura & 20 \\
\hline C & Explotación de minas y canteras & 20 \\
\hline D & Industrias manufactureras & 156 \\
\hline F & Construcción & 13 \\
\hline
\end{tabular}




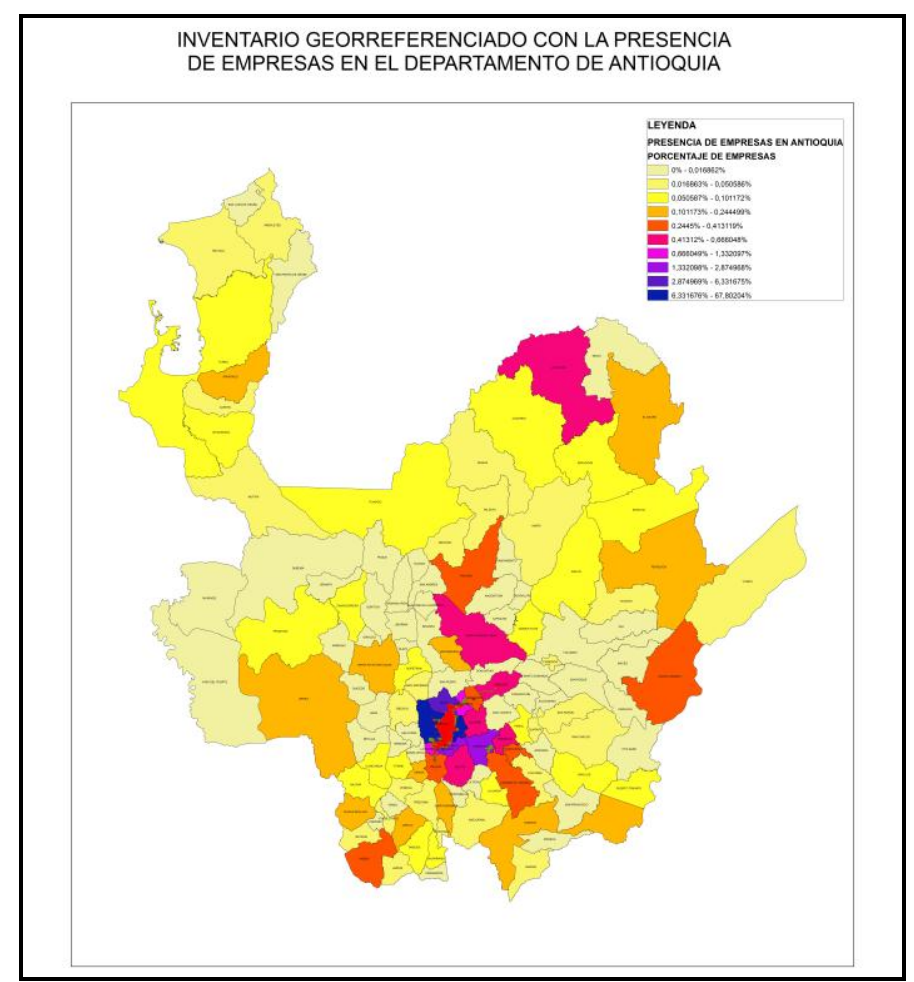

Fig 1. Inventario Georreferenciado con la presencia de Empresas en el Departamento de Antioquia (Elaboración propia)

Tabla 9. Sectores seleccionados por subregión

\begin{tabular}{|c|c|c|c|c|c|c|c|c|}
\hline \multirow[b]{2}{*}{ Actividad } & \multirow[b]{2}{*}{ Sector } & \multicolumn{7}{|c|}{ Número de empresas por subregión } \\
\hline & & $\begin{array}{l}\text { Bajo } \\
\text { Cauca }\end{array}$ & Nordeste & Norte & Oriente & Suroeste & Urabá & $\begin{array}{c}\text { Valle } \\
\text { de } \\
\text { Aburrá }\end{array}$ \\
\hline $\begin{array}{l}\text { Agricultura } \\
\text { (A) }\end{array}$ & $\begin{array}{l}\text { Agricultura, } \\
\text { ganadería, caza y } \\
\text { actividades de } \\
\text { servicios conexas } \\
\text { (01) }\end{array}$ & & & & 109 & & 83 & 791 \\
\hline \multirow{3}{*}{ Minería (C) } & $\begin{array}{l}\text { Extracción de carbón, } \\
\text { carbón lignitico y } \\
\text { turba (10) }\end{array}$ & & & & & 24 & & \\
\hline & $\begin{array}{l}\text { Extracción de } \\
\text { minerales metalíferos } \\
\text { (13) }\end{array}$ & 153 & 197 & & & & & \\
\hline & $\begin{array}{l}\text { Explotación de } \\
\text { minerales no } \\
\text { metálicos (14) }\end{array}$ & & & & & 7 & & \\
\hline \multirow{5}{*}{$\begin{array}{l}\text { Manufactura } \\
\text { (D) }\end{array}$} & $\begin{array}{l}\text { elaboración de } \\
\text { productos } \\
\text { alimenticios y de } \\
\text { bebidas (15) }\end{array}$ & & & & 117 & & & 517 \\
\hline & $\begin{array}{l}\text { fabricación de } \\
\text { productos textiles } \\
\text { (17) }\end{array}$ & & & & & & & 757 \\
\hline & $\begin{array}{l}\text { fabricación de } \\
\text { prendas de vestir; } \\
\text { preparado y teñido de } \\
\text { pieles (18) }\end{array}$ & & & 178 & 94 & & & 3217 \\
\hline & $\begin{array}{l}\text { curtido y preparado } \\
\text { de cueros; } \\
\text { fabricación de } \\
\text { calzado; fabricación } \\
\text { de artículos de viaje, } \\
\text { maletas, bolsos de } \\
\text { (19) }\end{array}$ & & & & & & & 492 \\
\hline & $\begin{array}{l}\text { transformación de la } \\
\text { madera y fabricación } \\
\text { de productos de }\end{array}$ & & & & 71 & & & 340 \\
\hline
\end{tabular}




\begin{tabular}{|c|c|c|c|c|c|c|c|c|}
\hline \multirow[b]{2}{*}{ Actividad } & \multirow[b]{2}{*}{ Sector } & \multicolumn{7}{|c|}{ Número de empresas por subregión } \\
\hline & & $\begin{array}{l}\text { Bajo } \\
\text { Cauca }\end{array}$ & Nordeste & Norte & Oriente & Suroeste & Urabá & $\begin{array}{l}\text { Valle } \\
\text { de } \\
\text { Aburrá }\end{array}$ \\
\hline & $\begin{array}{l}\text { madera y de corcho, } \\
\text { excepto muebles; } \\
\text { fabricación }(20)\end{array}$ & & & & & & & \\
\hline & $\begin{array}{l}\text { actividades de } \\
\text { edición e impresión y } \\
\text { de reproducción de } \\
\text { grabaciones (22) }\end{array}$ & & & & & & & 861 \\
\hline & $\begin{array}{l}\text { fabricación de } \\
\text { sustancias y } \\
\text { productos químicos } \\
(24)\end{array}$ & & & & 35 & & & 495 \\
\hline & $\begin{array}{l}\text { fabricación de } \\
\text { productos de caucho } \\
\text { y de plástico (25) }\end{array}$ & & & & & & & 449 \\
\hline & $\begin{array}{l}\text { fabricación de } \\
\text { productos elaborados } \\
\text { de metal, excepto } \\
\text { maquinaria y equipo } \\
\text { (28) }\end{array}$ & & & & 72 & & & 748 \\
\hline & $\begin{array}{l}\text { fabricación de } \\
\text { maquinaria y equipo } \\
\text { ncp (29) }\end{array}$ & & & & & & & 366 \\
\hline & $\begin{array}{l}\text { fabricación de } \\
\text { muebles; industrias } \\
\text { manufactureras ncp } \\
\text { (36) }\end{array}$ & & & & 120 & & & 959 \\
\hline $\begin{array}{l}\text { Construcción } \\
\text { (F) }\end{array}$ & Construcción (45) & & & & & & & 2332 \\
\hline Total & & 153 & 197 & 178 & 618 & 31 & 83 & 12324 \\
\hline
\end{tabular}

\subsection{Residuos generados en las empresas encuestadas}

\subsubsection{Actividad económica agricultura, ganadería, caza y silvicultura}

Los principales residuos de esta actividad son fracciones de cultivos que no constituyen la cosecha propiamente dicha y aquella parte de la cosecha que no cumple con los requisitos de calidad mínima para ser comercializada. De forma similar, los restos de poda de los cultivos leñosos deben ser considerados asimismo residuos agrícolas estrictos. En la ganadería los principales residuos son los purines, mezcla de orina y estiércol de animales [8], [9].

Se detectaron 6 clases de residuos, tres de los cuales no registraron cantidad, de los restantes son los residuos de tejido vegetal los más abundantes con un $89 \%$ del total. No obstante se considera que los datos obtenidos son insuficientes para realizar un mejor análisis. En la Fig. 2 se muestran los residuos de esta actividad con la siguiente nomenclatura; R1: Residuos de tejidos vegetales, R2: Residuos agroquímicos, R3: Residuos de tejidos de animales, R4: Residuos plásticos (excepto envases), R5: Lodos de lavado y limpieza, R6: Residuos no especificados en otra categoría, R7: llantas, R8: RAEES, R9: Heces animales, orina y estiércol (sin reporte)

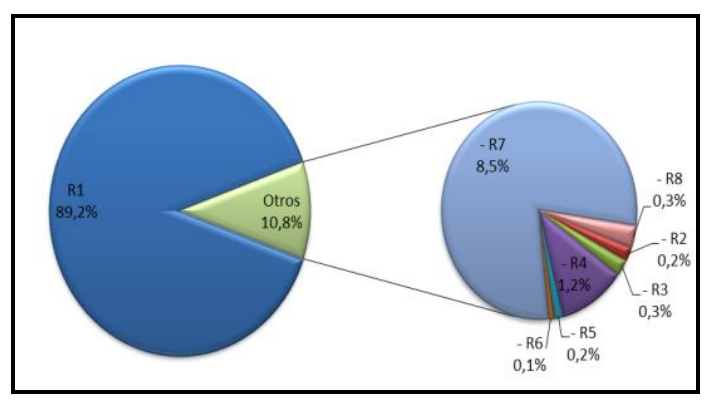

Fig. 2. Distribución de los residuos en la actividad económica Agricultura, ganadería, caza y silvicultura

\subsubsection{Actividad económica explotación de minas y canteras}

La industria minera representa uno de los sectores industriales con mayor capacidad de generación de residuos, con el agravante de que muchos de los estériles se hallan fuertemente contaminados por los productos químicos usados en el tratamiento in situ de los materiales explotados [8].

Para esta actividad se reportaron 21 clases de residuos, de ellos, 5 no presentaban reporte de cantidad. Los residuo que predominan en esta actividad son otros estériles que contienen sustancias peligrosas ocupando un $93.5 \%$ del total de los residuos. En la Fig. 3 , se muestran los residuos más representativos, con la siguiente nomenclatura. R1: Otros estériles que contienen sustancias peligrosas, R2: Residuos de polvo y arenilla, R3: Metales, R4: Estériles que generan ácido procedentes de la transformación de sulfuros, R5: Tiesto Reutilizado 100\%, R6: Estériles, R7: Aceite, 
R8: materiales contaminados con hidrocarburos, R9: Aceite usado, R10: grasa.

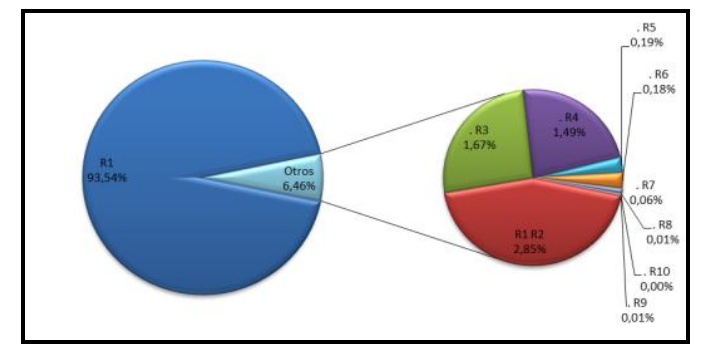

Fig. 3. Distribución de los residuos en la actividad económica explotación de minas y canteras

\subsubsection{Actividad económica manufactura}

Esta es la actividad económica que incluye más sectores productivos, presentando mayor diversidad de residuos; aunque las empresas que respondieron la encuesta fueron pocas (5\%), se registraron 75 tipos de residuos generados, para un total de 1.349.464,25 ton/año, de ellos quienes presentan una mayor proporción son: Polvillo (89 \%) y Metales (3,1\%). En la Fig. 4 se muestran los residuos más representativos con la siguiente nomenclatura; R1: Polvillo, R2: Metales, R3: Plástico, R4: Papel y cartón, R5: Escombros, R6: vidrio, R7: Carnazas y serrajes de encalado, R8: Residuos orgánicos, R9: Residuos de alimentos, R10: Desechos resultantes de la producción, la preparación y la utilización de disolventes orgánicos, R11: Residuos de fibras textiles procesadas, R12: Restos de textiles, R13: Polvo de tabaco, R14: Residuos de piel curtida (serrajes, rebajaduras, recortes y polvo de esmerilado) que contienen cromo, R15: Películas y papel fotográfico que no contienen plata ni compuestos de plata R16: Aserrín, virutas, recortes, madera, tableros de partículas.

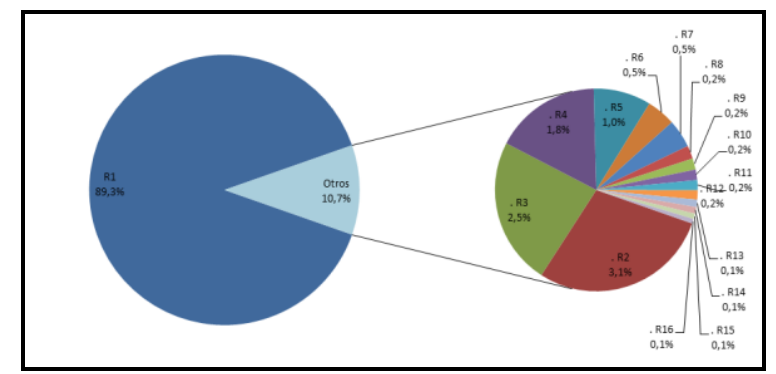

Fig. 4. Distribución de los residuos en la actividad económica manufactura

\subsubsection{Actividad económica construcción}

Los residuos procedentes de la construcción o demolición están compuestos en un gran porcentaje de materiales inertes. Una proporción muy pequeña de ellos contiene sustancias que hacen que se clasifiquen como peligrosos. La principal problemática ambiental asociada a ellos se deriva no sólo del creciente volumen de su generación, que supera algunas veces al de los residuos de origen doméstico, sino de su tratamiento [10].

En total se reportaron 30 tipos de residuos generados para un total de 4.991,16 ton/año, de ellos los que presentan un mayor porcentaje son la tierra y los escombros con un $50 \%$ del total de los residuos, en la Fig. 5 se muestran los principales residuos con la siguiente nomenclatura; R1: Hierro y acero, R2: Materiales de construcción, R3: Tejas y materiales cerámicos, R4: Madera, R5: Plástico, R6: Cobre, bronce, latón, R7:Tierra y piedras que contienen sustancias peligrosas, R8: Papel y cartón, R9: Retales de piedras para enchapes y lodos por corte refrigerado con agua, R10: Papel de oficina, R11: Cajas de cartón, R12: Papel periódico, R13: Fibra de vidrio, R14: Refrigerante freón 22, R15: Hormigón, R16: Ladrillos, R17: Cables, R18: Residuos de construcción y demolición mezclados o fracciones separadas que contienen sustancias peligrosas, R19: PVC, R20: Cartón, R21: Tierra y escombros, R22: Metales, R23: Vidrio, R24: Aluminio, R25: Metales mezclado, R26: Trapos impregnados de aceite y disolvente, R27: Aceites usados contaminados, R28: laminas usadas de fibra, R29: refrigerantes (su estado es gaseoso), R30: Lodos de drenaje que contienen sustancias peligrosas.

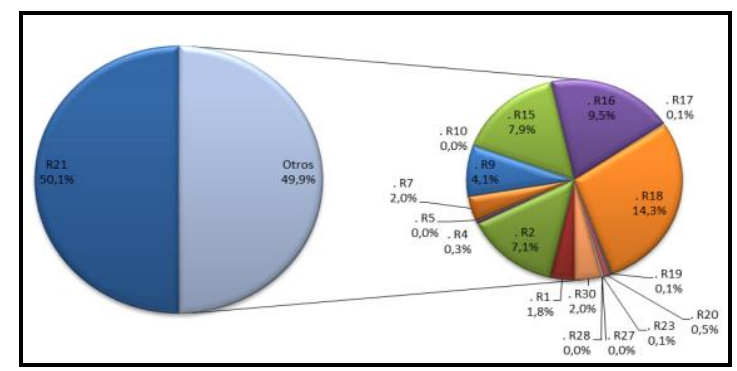

Fig. 5. Distribución residuos en la actividad económica construcción

\subsection{Evaluación del impacto ambiental}

Luego de realizar la evaluación a todos los residuos reportados por las diferentes empresas, de acuerdo con la metodología utilizada para la evaluación del impacto ambiental, se encontraron 11 residuos con un mayor impacto al ambiente, de los cuales se resaltan los estériles con sustancias peligrosas, provenientes del proceso de beneficio del oro, los cuales presentan un impacto de -496 con una importancia crítica según la valoración de impacto ambiental, seguido por residuos de fibras textiles procesadas y estériles de la transformación de sulfuros con un impacto de -456 y -442 , respectivamente y con una importancia severa de acuerdo a la valoración del impacto. Aunque en el proyecto solo se contemplan residuos sólidos, en la valoración ambiental se tuvieron en cuenta los residuos líquidos reportados por las empresas, como es el caso de los disolventes orgánicos que tienen una importancia severa por presentar una valoración de -432. (Tabla 10).

Teniendo en cuenta la valoración del impacto ambiental en el momento de priorizar un residuo para investigación, son estos 11 residuos los que presentarían la más alta prioridad, sin embargo, es importante tener en cuenta otros parámetros como, disponibilidad del residuo para investigación, deseo de las empresas en participar en el proceso investigativo y alternativas tecnológicas, a la hora de iniciar una investigación con un residuo en particular. 
Tabla 10. Residuos con una mayor valoración de impacto.

\begin{tabular}{|l|c|}
\hline \multicolumn{1}{|c|}{ Tipo de Residuo } & Valor del Impacto \\
\hline Estériles con sustancias peligrosas & -496 \\
\hline Residuos de fibras textiles procesadas & -456 \\
\hline Estériles de la transformación de sulfuros & -442 \\
\hline Escombros con sustancias peligrosas & -432 \\
\hline Disolventes orgánicos & -432 \\
\hline Aceites usados & -390 \\
\hline Lodos del tratamiento de aguas, que contienen cromo & -380 \\
\hline Residuos fotográficos sin plata & -380 \\
\hline Residuos de curtido de piel que contienen cromo & -372 \\
\hline Polvo de Tabaco & -370 \\
\hline Carnazas y cerrajes de encalado & -364 \\
\hline
\end{tabular}

\section{CONCLUSIONES}

Durante la primera fase del proyecto se identificaron cuatro actividades económicas: agricultura, ganadería, caza y silvicultura; Explotación de minas y canteras; Industrias manufactureras y Construcción, en siete de las nueve subregiones del departamento de Antioquia: Bajo Cauca, Nordeste, Norte, Oriente, Suroeste, Urabá y Valle de Aburrá; como las principales generadoras de residuos industriales.

En estas subregiones se aplicó la encuesta a un total de 12.054 empresas, con una respuesta de 519 de ellas, lo que equivale al 4,3\%, de las cuales el $0,9 \%$ corresponden a la actividad Agricultura, ganadería, caza y silvicultura, el $18,8 \%$ a la actividad explotación de minas y canteras, el $4,5 \%$ a la actividad manufacturera y el $1,9 \%$ a la actividad de la construcción. Este bajo porcentaje puede indicar una falta de compromiso del sector industrial con la búsqueda de soluciones para la problemática de los residuos, lo que se ve reflejado en una apatía a la hora de responder encuestas 0 se evidencia en el diligenciamiento incompleto de algunas de ellas o tal vez es necesario implementar nuevas estrategias de comunicación para mejorar la cantidad de encuestas diligenciadas.

Los resultados de las encuestas muestran que los residuos de mayor generación en el departamento de Antioquia son los residuos de tejidos vegetales generados en la actividad agrícola; los estériles con contenido de sustancias peligrosas en la actividad minera; el polvillo (residuo de la industria cementera), metales, plástico, papel y cartón provenientes de la industria manufacturera; la tierra y escombros, residuos de construcción y demolición mezclados o fracciones separadas que contienen sustancias peligrosas, ladrillos y hormigón de la actividad de la construcción.

La gran variedad de residuos generados en los sectores seleccionados demandó la aplicación de un método que permitiera la priorización de residuos a estudiar en una siguiente fase del proyecto, considerando varios criterios como el impacto, cantidad generada, cantidad de empresas generadoras, periodicidad en su generación, peligrosidad y manejo actual; dando como resultado que los estériles con sustancias peligrosas, los residuos de fibras textiles procesadas, los estériles de la transformación de sulfuros y los escombros con sustancias peligrosas, son los residuos más prioritarios a estudiar.

Aun con la poca respuesta del sector empresarial, se encontró una gran variedad de residuos, la mayoría de ellos sin un aprovechamiento lo que muestra la importancia de la continuidad del proyecto. Para ello se propone la divulgación de éste por medios escritos, electrónicos y revistas especializadas, además de investigar otras metodologías existentes para la realización de inventarios, para complementar la información obtenida.

El inventario de los residuos de la industria en el departamento de Antioquia es un proyecto de gran magnitud, que requiere de muchos recursos económicos y técnicos; para unos mejores resultados en cuanto a respuesta y vinculación de los generadores de residuos con el proyecto, se propone la continuidad de éste mediante proyectos focalizados por actividades económicas, lo que permitirá tener un mayor acercamiento a los sectores de interés, una mayor divulgación del proyecto a través de agremiaciones y posiblemente una mejor respuesta del sector industrial.

\section{AGRADECIMIENTOS}

Al programa expedición Antioquia 2013; a los grupos de investigación de la Universidad de Antioquia, Grupo de Ingeniería y Gestión Ambiental, GIGA, Grupo Procesos Fisicoquímicos Aplicados, PFA; al grupo de investigación de la universidad de San Buenaventura Grupo de Investigaciones en Modelamiento y Simulación Computacional, GIMSC y al grupo de investigación de la Universidad de Medellín Grupo de Investigaciones y Mediaciones Ambientales, GEMA. 


\section{REFERENCIAS}

[1] World Resources Institute. Millennium Ecosystem Assessment. Ecosystems and Human Well-being: Biodiversity Synthesis. Washington, DC., 2005.

[2] V. K. Muñóz \& O.A. Bedoya. "El papel de los residos sólidos, en la solición de problemas ambientales". Economía autónoma, Colombia, 2009.

[3] C. Bustos. "La problemática de los desechos sólidos". Online [Jun. 2009].

[4] Departamento Administrativo de Planeación. Anuario Estadístico de Antioquia, 2006.

[5] Departamento de Asuntos Económicos y Sociales División de Estadística, Naciones Unidas. Clasificación Industrial Internacional Uniforme de todas las actividades económicas - CIIU, (Revisión 3.1). 2005.
[6] V. Coneza. Guía metodológica para la evaluación ambiental. 2nd. ed. Madrid: Editorial Mundi-prensa, 1995.

[7] J. Arboleda. "Una propuesta para la identificación y evaluación de impactos ambientales". Crónica Forestal y del Medio Ambiente 9, pp.71-81, 1994.

[8] X. E. Castells. Reciclaje de residuos industriales: residuos sólidos urbanos y fangos de depuradora. España: Diaz de Santos, 2009. p. 1295.

[9] A. V. Bridgwater \& C. J. Mumford. Waste Recycling and pollution control Handbook, New York: Van Nostrand Reinhold, 1979. p. 706.

[10] N. Quaranta, M. Caligaris, H. López \& M. Unsen. "II Simposio Iberoamericano de Ingeniería de Residuos". Barranquilla, Septiembre, 2009. 